\title{
Organic chemistry in planetary satellites of gas giants and implications for habitability
}

\author{
Athena Coustenis \\ Laboratoire d'Etudes Spatiales et d'Instrumentation en Astrophysique (LESIA), \\ Observatoire de Paris, PSL-Research Univ., CNRS, Univ. Paris 06, Sorbonne Univ., Univ. \\ Paris-Diderot, Sorbonne Paris-Cité, 5, place Jules Janssen, 92195 Meudon Cedex, France \\ email: athena.coustenis@obspm.fr
}

\begin{abstract}
We look at the icy moons in our outer solar system in which we find organics and the possibility for habitabile conditions therein.
\end{abstract}

Keywords. planets and satellites: formation, methods: data analysis

\section{Introduction}

The icy satellites of the outer solar system present a variety of chemical compositions where initial complements of ices, minerals, and elements provided during formation have been subjected to various internal and external processes. Additional material has been gained via cometary and meteoritic infall, transfer of material between satellites, and magnetospheric interactions. Exchanges also occur between the surface and the interior.

Organic compounds have been detected on several of our Solar System's natural satellites around the gas giant planets, and each object has unique characteristics with regard to organic chemistry. Jupiter's Europa, Ganymede and Callisto show evidence of undersurface layers of liquid water that offer potentially uniquely interesting environment for organic synthesis. Spectra obtained by Galileo show absorption bands indicative of C-H and $\mathrm{C}-\mathrm{N}$ organic compounds. Potential organics on the large Galilean moons include $\mathrm{CO} 2$, carbonic acid and different kinds of carbonates, hydrocarbons and nitriles. Titan and Enceladus around Saturn have also been recognized to possess organic components.

\section{Habitability definitions}

Our perception of habitability in the solar system is evolving. The definition of habitability is the suitability for living in or on. Planetary habitability is the measure of a planet's or a natural satellite's potential to develop and sustain life. Life may develop directly on a planet or satellite or be transferred to it from another body (panspermia). As the existence of life beyond Earth is currently uncertain, planetary habitability is largely an extrapolation of conditions on Earth and the characteristics of the Sun and Solar System. Research and theory in this regard is a component of planetary science and of astrobiology. Space agencies have defined the principal habitability criteria as "extended regions of liquid water, conditions favorable for the assembly of complex organic molecules, and energy sources to sustain metabolism over long periods of time".

In the work by Lammer et al. (2009), several classes of habitats were defined :

- Class I habitats represent bodies on which stellar and geophysical conditions allow Earth-analogue planets to evolve so that complex multi-cellular life forms may originate.

- Class II habitats are bodies on which life may emerge but due to unfavorable stellar and geophysical conditions, evolve toward barren Venus- or Mars-type worlds. 
- Class III habitats are planetary bodies where subsurface water oceans may exist such as on Europa, which interact directly with a silicate-rich core, while

- Class IV habitats have liquid water layers between two ice layers, or liquids above ice.

The Habitable Zone (HZ) is the scientific term for the region around a star within which it is theoretically possible for a planet with sufficient atmospheric pressure to maintain liquid water on its surface. The HZ theory suggests that it is possible for a planetary mass object in the habitable zone to sustain liquid water on its surface. However it does not follow that liquid water must exist on the surface of such a planet. Some considerations relate to climate, rather than temperature, including the nature of the star and the orbit of the planet which can work for or against the presence of surface water on the planet. Along with these basic considerations, there are many additional criteria for planetary habitability: low gravity (Mars), runaway greenhouse warming (Venus), etc. Space weather, in particular stellar radiation and stellar variation, can significantly affect the ability of planets within the habitable zone to retain surface water. Venus and Mars are examples of planets that may have experienced significant and relatively rapid loss of surface water. A planet may require an intrinsic defense mechanism against the effects of space weather, such as a combination of magnetosphere, atmosphere, geological and geophysical cycles to sustain stable bodies water on the surface. The Earth possesses such a combination of defenses and atmospheric replenishing occurs via volcanism and processes such as the carbon cycle and biological processes. Other processes have been observed on other planets, such as exchange of atmospheric materials between Enceladus and Saturn through geysers as well as between Io and the other moons of Jupiter. Other theoretical processes include outgassing and cryovolcanism.

The appropriate spectral range for "HabStars" is presently considered to be "early F" or "G", to "mid-K" temperatures of 4000-7000 K; the Sun, a G2 star, is well within these bounds). The outer solar system is potentially a huge reservoir of water in the form of ice (Coustenis et al. 2010, Coustenis \& Encrenaz 2013). With the exception of Titan, the icy moons with possible subsurface oceans and/or activity reside in giant planet magnetospheres, but Enceladus and Triton are not in the magnetospheric section with the most extreme surface irradiation harmful to organics.

\section{Icy moons as potential habitats}

Icy moons where the water percentage is considerable (almost $50 \%$ in weight) are described as waterworlds. Two of the five bodies of planetary families already identified in the galaxy this far are represented by Galilean moons. The five families range from Mercury-like to Jupiter-like bodies depending on the amount of silicates, followed by water, and finally $\mathrm{H}$ and $\mathrm{He}$ which are progressively increased in these bodies. By 2030, we don't know yet how many thousands of planets will have been discovered, but what we can affirm is that only two families will have been properly characterized, namely Mercury-like (thanks to Messenger and Bepi-Colombo), and Earth-like of course. Hence the need for a new mission which by thorough exploration of Ganymede and of the other satellites would characterize the best example of an entire class of planets, namely the waterworlds! ESA's JUICE mission will attempt to answer some of these questions.

Galilean satellites. Among the Galilean moons we find several representatives of the two waterworld classes. Ganymede (class IV) is the biggest moon of the solar system, bigger than our own moon and even Mercury. Gravity data point to a fully differentiated structure such as the one illustrated on this screen. From top to bottom, it is believed to be layered as follows : It possesses a very tenuous atmosphere, an hydrosphere which 
may be at least $500 \mathrm{~km}$ thick (meaning $50 \% \mathrm{wt}$ ), a silicate mantle and an iron core. A liquid layer up to $100 \mathrm{~km}$ thick is trapped between the icy crust on top and a layer composed of high pressure ices, which do not exist in natural environments on the Earth. It is one of three solid bodies which possess an intrinsic or dynamo magnetic field (along with Mercury and the Earth) and this magnetic field is in turn embedded in the Jovian magnetic field. Finally, it also has an induced magnetic field, generated by currents flowing in a conducting liquid, the best proof of the existence of the deep ocean.

Callisto is very similar to Ganymede, except that it is not fully differentiated and that it does not have an intrinsic field. Its surface is very very old and heavily cratered, and also it possesses a deep ocean similar to Ganymede.

Europa is layered in a same way. We think that it possesses a similar metallic core and a silicate layer, about the same size as Ganymede's, but a thinner hydrosphere, no more than $100 \mathrm{~km}$ thick. The big difference here is that the liquid layer is almost certainly in direct contact with the silicates. Class III habitats like Europa, where subsurface oceans are in contact with silicates on the sea-floor, open the question of where the building blocks for life come from. By assuming that the organic material necessary to start life is supplied by impact of meteorites and comets and by precipitation of interplanetary dust, this material impacting on the surface has to find its way into the subsurface oceans. Also this material has to reach meaningful concentrations somewhere in the ocean, which is hard to imagine in a large planet-wide subsurface ocean. However, one should keep in mind that synthesis of organic material by either Fischer-Tropsch reactions (which convert carbon monoxide and hydrogen into hydrocarbons) or catalytic cycles are possible under the high pressure/high temperature conditions occurring at deep-sea vents. Indeed, in such conditions on Earth, reduced radicals in hot liquid can provide the energy for the sustainability of some living organisms, although not to power them.

Class IV habitats and exoplanets where a water ocean is in contact with a thick ice layer offer a much better situation for the influx of organic material from outside the planet compared to bodies like the Jovian satellites Ganymede or Callisto. The main problem encountered in Class IV habitats is, however, much more severe: that of the concentration of the necessary ingredients for life. A planet whose surface is completely covered in water several kilometers deep with nothing to act as a concentrating "sponge" for organic chemistry is probably too vast for any two or more interesting molecules to meet. While a sea/ice system could in theory provide such a means of concentrating life's ingredients, most likely the starting concentrations needed for a system like that may be difficult to reach in addition to the quite specific temperature conditions needed.

Concerning the energy sources, the presence of a $\mathrm{H}_{2} \mathrm{O}_{2}$ oxidizing chemistry at the surface, due to the radiolysis of ice by radiation belt particles, and of a reducing chemistry at the ocean floor if hydrothermal activity exists, opens the possibility for the existence of a redox couple acting at the scale of the ocean. The initial redox chemical disequilibrium upon which all living things depend for energy, arises from the separation of reducing and oxidizing chemical species in the first place. But this works only if oxidants from the surface can feed the ocean via a (hypothesized) partly permeable icy crust.

Ganymede and Europa are two archetypes of two classes of habitable planets that will be explored by JUICE (Grasset et al. 2013):

- The conditions existing at the bottom of the ocean on Europa are probably similar to those of our oceans where life has developed. No space mission can explore these deep domains, but at least, it is possible with JUICE to explore the recently active zones at the surface. From this study JUICE will constrain the likelihood of such habitats and pave the way for a future lander mission devoted to the search for past and present life.

- On Ganymede, it is not certain that the exchange processes between the silicates 
and the liquid layer, are possible. JUICE will answer this important question because it concerns an entire family of planets whose occurrence in the galaxy certainly far surpasses the Earth-like planets since they occupy the stellar zone beyond the snow line. Depending on the results of the mission, the probability of having habitable worlds in the universe (one of the key parameters of the Drake formula) could be significantly changed.

Saturnian moons. In the Saturnian system, Titan is a natural chemical factory, where the mother molecules N2 (at about 95\%), methane (at 1.5-5\%) and hydrogen (at 0.1\%) produce a host of hydrocarbons and nitriles. The Cassini-Huygens mission has shown Titan to be indeed very rich in organic molecules, which are formed in the upper atmosphere and then deposited on the surface. Some of these species are of prebiotic importance, such as $\mathrm{C}_{6} \mathrm{H}_{6}, \mathrm{HC}_{3} \mathrm{~N}$ and $\mathrm{HCN}$. Titan's surface displays unique geomorphological features while it probably overlies an internal liquid water ocean. The organic deposits, in coming into contact with the liquid water in the underground could possibly undergo an aqueous chemistry that could replicate aspects of life's origins. While being a unique object of investigation on its own, Titan provides a good analogue as a natural laboratory in which chemical and physical processes can be studied on a planetary scale and help us understand early chemical evolution in the primordial atmosphere on Earth. The study of the gases and aerosols in Titan's atmosphere gives hints on the chemical evolution on the early Earth because prebiotic evolution on Earth involved atmospheric particles and organic particles that are present in Titan's atmosphere and also because chemical evolution may have been enhanced by the process of aerosol formation and growth.

The surface of Titan appears at first glance to be an unlikely location for life, at least terrestrial-type life. There are photochemically derived sources of free energy on Titan's surface which could support life, which would have to be an exotic type of life using liquid hydrocarbons as solvents (Committee on the Origin and Evolution of Life 2007). Terrestrial bacteria can in fact derive their energy and carbon needs by "eating" tholins. In this sense, a methane-rich atmosphere may act as a "poor-planet's photosynthesis", providing a means to capture the free energy from ultraviolet light and make it available for metabolic reactions. Benner et al. (2004) have speculated that a form of life, or at a minimum a kind of organized chemical system, can be sustained in liquid hydrocarbons known to be stable on Titan's surface. And McKay and Smith (2005) predicted that Titanian life if it existed on the surface, would consume near-surface hydrogen and that this might be detectable.

Enceladus, a smaller moon of Saturn, ejects large amounts of water and organics in the space from plumes currently located in its southern pole. The implied requirement for liquid water reservoirs under its surface, significantly broadens the diversity of solar system environments which could be habitable worlds.

Enceladus and Titan currently represent the most distant potential under-surface liquid water reservoirs in the solar system. We will certainly also need future missions to study these moons in the Saturnian system.

\section{References}

Benner, S. A., Ricardo, A., \& Carrigan, M. A. 2004, Current Opinion in Chemical Biology, 8, 672

Coustenis, A., Tokano, T., Burger, M. H., Cassidy, T. A., Lopes, R. M., Lorenz, R. D., Retherford, K. D., \& Schubert, G. 2010, Space Sci. Rev., 153, 155

Coustenis, A. \& Encrenaz, T. 2013, in: Life Beyond Earth (Cambridge Univ. Press), ISBN: 9781107026179 
Grasset, O., Dougherty, M. K., Coustenis, A., Bunce, E. J., Erd, C., Titov, D., Blanc, M., Coates, A., Drossart, P., Fletcher, L. N., Hussmann, H., Jaumann, R., Krupp, N., Lebreton, J.-P., Prieto-Ballesteros, O., Tortora, P., Tosi, F., \& Van Hoolst, T. 2013, Pla. Space Sci., 78, 1 Lammer, H., Bredehoft, J. H., Coustenis, A., Khodachenko, M. L., Kaltenegger, L., Grasset, O., Prieur, D., Raulin, F., Ehrenfreund, P., Yamauchi, M., Wahlund, J.-E., Griesmeier, J.-M., Stangl, G., Cockell, C. S., Kulikov, Yu.N., Grenfell, J. L., \& Rauer, H. 2009, A \& A, 17, 181

McKay, C. P. \& Smith, H. D. 2005, Icarus, 178, 274 